\title{
A NEW ROOF MODEL ON RANDOMLY PLACED BUILDINGS IN MOBILE COMMUNICATION
}

\author{
N. Altin and E. Yazgan \\ Electrical \& Electronics Engineering Department \\ Hacettepe University \\ 06532, Beytepe, Ankara, Türkiye
}

\begin{abstract}
Multiple diffraction propagation path loss due to the successively located different shaped building roofs are important for mobile communication. In the literature, building roofs are considered as wedge shaped structures. In this paper, building roof is modelled with a more realistic structure and the propagation path loss between the transmitting antenna and the receiving antenna is calculated.
\end{abstract}

\section{INTRODUCTION}

The extended uniform geometrical theory of diffraction has been applied to the problem of investigating the effects of intervening terrain on the propagation path of high frequency electromagnetic waves $[4,8]$. The new diffraction coefficient was applied to calculate the diffraction due to the perfectly conducting roofs and rough lossy roofs. Also the path loss predictions due to the various shapes of terrain profiles with varying conductivity, permittivity and surface roughness were obtained. Successful results have been obtained for frequencies between $100 \mathrm{MHz}$ and $10 \mathrm{GHz}$. In this work, surface waves haven't been taken into consideration [4].

Another important problem, being of the second roof is in the shadow region boundary of the diffracted fields of the first roof, discontinuity occurrence is seen in the results obtained for the electric field strength. Heuristic slope diffraction correction term has been used for removing this discontinuity. The slope diffraction term provides continuity on both perfectly conducting roofs and rough lossy roofs for both polarizations. For grazing incidence between roof vertices and lossy roof, diffraction coefficient is multiplied by a special gain factor [5]. 
In this study, the comparison of the diffracted fields for the perfectly conducting and rough lossy roofs are given for both polarizations by depending on interior angles of building roof changes. The chosen geometry is more realistic one for the exact calculation of the path loss due to roofs of building in the urbans.

\section{FORMULATION}

In the formulation, three kinds of rays have been taken into formulation in the new uniform geometrical theory of diffraction [2]. These rays are

a) Direct ray

b) Reflected rays from the roofs surface

c) Diffracted rays from roofs vertices of roofs.

The expressions of rays as shown in Figure 1.

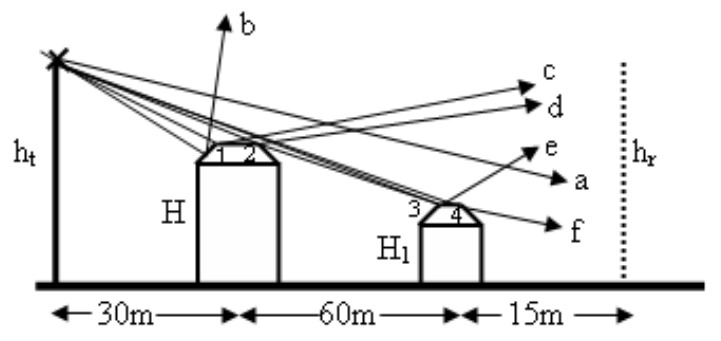

Figure 1. Propagation model between the transmitting and the receiving antenna.

a) Direct ray: This ray is the ray between the transmitting antenna and the receiving antenna. The expression of direct ray is given as below

$$
E_{i}=\frac{e^{-i k s}}{k s} U_{1}
$$

Where $U_{1}$ is the unit step function related to direct ray. $s$ is the total path between the transmitting antenna and the receiving antenna.

b) Reflected rays from roof surface: This ray is the ray that arrives to the receiving antenna form the transmitting antenna by reflecting from the roof surface. Reflected ray from the roof surface is given as below

$$
E_{r}=R \frac{e^{-i k\left(s_{1}+s_{2}\right)}}{k\left(s_{1}+s_{2}\right)} U_{2}
$$

Where $U_{2}$ is the unit step function related to reflected rays region. $s_{1}$ is the distance from the transmitting antenna to the reflection point 
on the roof surface, $s_{2}$ is the distance from the reflection point on the roof surface to the receiving antenna. $R$ is the reflection coefficient from roof surface for the appropriate polarization. In the perfectly conducting roof surface is taken -1 for horizontal polarization and +1 for vertical polarization. In the rough and lossy roof, the reflection coefficient is given as

$$
R=R_{s} \cdot \rho
$$

Where $R_{s}$ is the plane wave reflection coefficient, $\rho$ is the surface roughness attenuation factor. Here

$$
R_{s}=\frac{\sin \varphi-\sqrt{\hat{\varepsilon}_{r}-\cos ^{2} \varphi}}{\sin \varphi+\sqrt{\hat{\varepsilon}_{r}-\cos ^{2} \varphi}}, \quad R_{s}=\frac{\hat{\varepsilon}_{r} \sin \varphi-\sqrt{\hat{\varepsilon}_{r}-\cos ^{2} \varphi}}{\hat{\varepsilon}_{r} \sin \varphi+\sqrt{\hat{\varepsilon}_{r}-\cos ^{2} \varphi}}
$$

are the reflection coefficients for horizontal and vertical polarizations, respectively.

$$
\rho=e^{-\delta^{2} / 2}, \quad \delta=\frac{4 \pi \Delta h}{\lambda} \sin \varphi
$$

With $\Delta h$ is the standard deviation for the normal distribution of the local surface roughness and $\lambda$ is the wavelength.

$$
\hat{\varepsilon}_{r}=\varepsilon_{r}-i \sigma / \omega \varepsilon_{0}
$$

$\hat{\varepsilon}_{r}$ is the complex permittivity and $\sigma$ is the conductivity. Diffraction coefficient is multiplied by a special factor for the grazing incidence between vertices of the roof those have finite conductivity [4].

c) Diffracted ray: This ray is the ray that is diffracted by striking vertices of roof and reaching the receiving antenna. Diffracted ray from vertices of the roof is expressed by

$$
E_{1}^{d}=E_{2}^{i}=E_{1}^{i} D_{1}\left(\phi_{1}, \phi_{1}^{\prime}\right) \sqrt{\frac{s^{\prime}}{s^{\prime \prime}\left(s^{\prime}+s^{\prime \prime}\right)}} e^{-i k s^{\prime \prime}} U_{3}
$$

Where $s^{\prime}=\left|\bar{s}^{\prime}\right|$ is the distance from transmitting antenna to the corner of the roof. $s^{\prime \prime}=\left|\bar{s}^{\prime \prime}\right|$ is the distance from the corner of the roof to the receiving antenna. $\phi_{1}^{\prime}$ is the angle between $s^{\prime}$ and left surface of the roof. $\phi_{1}$ is the angle between $s^{\prime \prime}$ and the left surface of the roof. $D_{1}$ is the diffraction coefficient for the uniform geometrical theory of diffraction [1]. Here $U_{3}$ is the unit step function related to diffracted ray region.

d) Doubly diffracted ray: This ray is the ray that is diffracted again form the second tip of the roof and reaching to the receiver antenna after diffracting from first tip of roof. Slope diffraction term 
is added for this ray. The expression for doubly diffracted field is given by

$$
\begin{aligned}
E_{3}^{i} & =E_{2}^{d} \\
& =\left[E_{2}^{i} D_{2}\left(\phi_{2}, \phi_{2}^{\prime}\right)+\frac{1}{2 i k} \frac{\partial D_{2}\left(\phi_{2}, \phi_{2}^{\prime}\right)}{\partial \phi^{\prime}} \frac{\partial E_{2}^{i}}{\partial u_{2}}\right] \sqrt{\frac{s^{\prime}+s^{\prime \prime}}{s^{\prime \prime \prime}\left(s^{\prime}+s^{\prime \prime}+s^{\prime \prime \prime}\right)}} e^{-i k s^{\prime \prime \prime}} U_{4}
\end{aligned}
$$

Where $s^{\prime}=\left|\bar{s}^{\prime}\right|$ is the distance from transmitting antenna to the corner of the roof. $s^{\prime \prime}=\left|\bar{s}^{\prime \prime}\right|$ is the distance between two tip points of the roof. $s^{\prime \prime \prime}=\left|\bar{s}^{\prime \prime \prime}\right|$ is the distance from the second tip of the roof to the receiving antenna. $\phi_{2}^{\prime}$ is the angle between $s^{\prime \prime}$ and left surface of the roof. $\phi_{2}$ is the angle between $s^{\prime \prime \prime}$ and left surface of the roof. $D_{2}$ is the diffraction coefficient in the uniform geometrical theory of diffraction in [1]. Open expression of the slope diffraction term is given in [5]. Here $U_{4}$ is the unit step function related to diffracted ray region.

e) Triply diffracted ray: This ray consists of diffracted-diffracted ray and addition diffraction of this ray from the single tip of the roof. Explanation of the doubly diffracted ray is given above is valid also for this case. Diffracted-diffracted-diffracted field will have the following form.

$$
E_{4}^{i}=E_{3}^{d}=E_{3}^{i} D_{3}\left(\phi_{3}, \phi_{3}^{\prime}\right) \sqrt{\frac{s^{\prime}+s^{\prime \prime}+s^{\prime \prime \prime}}{s^{\iota v}\left(s^{\iota v}+s^{\prime}+s^{\prime \prime}+s^{\prime \prime \prime}\right)}} e^{-i k s^{\iota v}} U_{5}
$$

Here $U_{5}$ is the unit step function related to triply diffracted field region.

f) Four times diffracted ray: This ray consists of four successive diffraction from the tips of the roof. Explanation that is given above is valid also for this situation. $E_{4}^{d}$ is given by

$E_{4}^{d}=\left[E_{4}^{i} D_{4}\left(\phi_{4}, \phi_{4}^{\prime}\right)+\frac{1}{2 i k} \frac{\partial D_{4}\left(\phi_{4}, \phi_{4}^{\prime}\right)}{\partial \phi^{\prime}} \frac{\partial E_{4}^{i}}{\partial u_{4}}\right] \sqrt{\frac{s^{\iota v}+s^{\prime}+s^{\prime \prime}+s^{\prime \prime \prime}}{s\left(s+s^{\iota v}+s^{\prime}+s^{\prime \prime}+s^{\prime \prime \prime}\right)}} e^{-i k s}$

Total field that is the sum of these rays can be found as below. 14 different types of rays are examined in the formulation with the consideration of various points due to their special features.

$$
\begin{aligned}
E_{\text {toplam }}= & E_{i}+E_{r}+E_{k 1}+E_{k 2}+E_{k 3}+E_{k 4}+E_{k 1 k 2}+E_{k 2 k 3} \\
& +E_{k 2 k 4}+E_{k 3 k 4}+E_{k 2 k 3 k 4}+E_{k 1 k 2 k 4}+E_{k 1 k 2 k 3}+E_{k 1 k 2 k 3 k 4}
\end{aligned}
$$

Here, subindexes; $i$ represents incident, $r$ represents reflected and $k$ represents the singly and doubly diffracted fields. 
Progress In Electromagnetics Research C, Vol. 3, 2008

\section{THE CALCULATION OF PROPAGATION PATH LOSS WITH HEURISTIC SLOPTE DIFFRACTION METHOD THAT IS APPLIED CONSECUTIVE ROOFS WITH VARIOUS ANGLES}

Results can be observed as given in the Figure 2 and Figure 3 by calculating the diffracted field loss between the transmitting and the receiving antennas with the aid of the total field which is expressed by above formulas for the geometry seen in Figure 1. Here receiver antenna height is considered as a parameter.

Slope diffraction correction term corrects the discontinuity problem that is caused by a perfectly conducting roof with a horizontally polarized incident field and a rough lossy roof for both polarizations. In this study, reflections from the ground weren't included so as to show the effect of the diffracted fields in the path loss computations clearly. These reflections must be included in any actual path loss computations.

Rough and lossy parameters are given as relative permittivity 15 , conductivity $0.012 \mathrm{~S} / \mathrm{m}$ and local surface roughness $23 \mathrm{~cm}$. The comparison of the losses those are caused by the diffracted fields on the perfectly conducting or rough and lossy roofs for both polarizations are given by changing the angles $\left(95^{\circ}-115^{\circ}\right)$ between the tips of the building roofs in Figures 2 and 3.

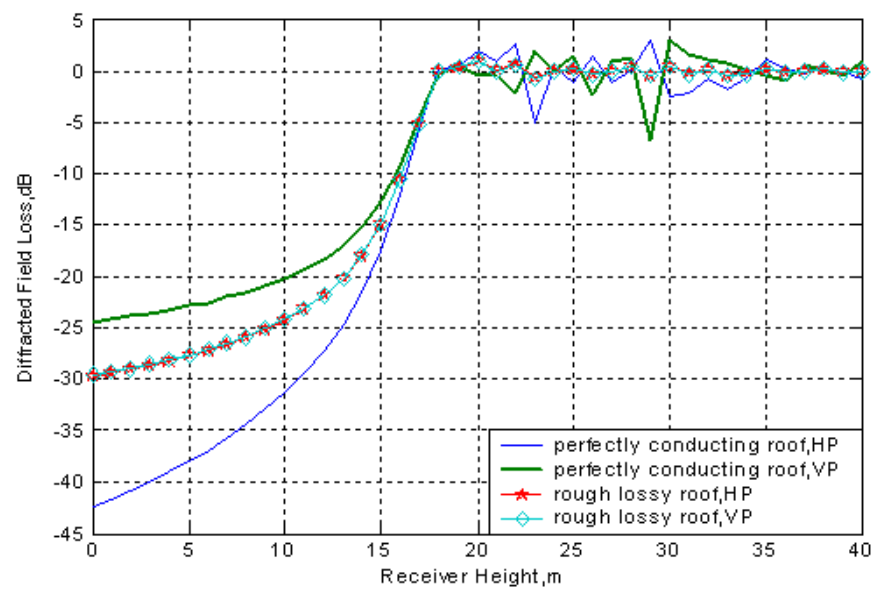

Figure 2. Diffracted field loss while angles of building roof vertices are $95^{\circ}$. Frequency is $900 \mathrm{MHz}, 1$. building height $30 \mathrm{~m}, 2$. building height $20 \mathrm{~m}$ and thickness $10 \mathrm{~m}$, height of transmitter antenna $\left(h_{t}\right) 45 \mathrm{~m}$. 


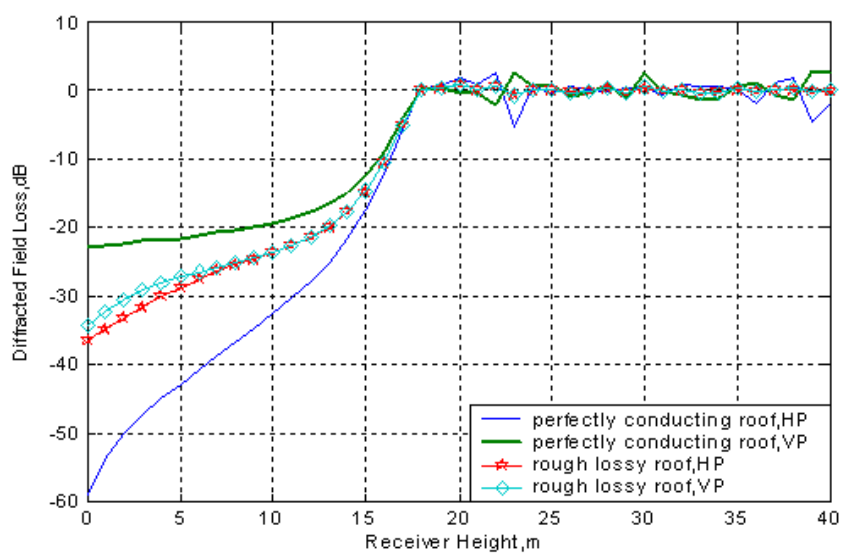

Figure 3. Diffracted field loss while angles of building roof vertices are $115^{\circ}$. Frequency is $900 \mathrm{MHz}, 1$. building height $30 \mathrm{~m}, 2$. building height $20 \mathrm{~m}$ and thickness $10 \mathrm{~m}$, height of transmitter antenna $\left(h_{t}\right) 45 \mathrm{~m}$.

Attenuation of the received waves is increased in the perfectly conducting roofs for horizontal polarization and in the rough and lossy roofs for both polarizations when the angle between the tips of the building roof is increased, as seen in Figures 2 and 3. There is no difference between horizontally and vertically polarizations for lossy surfaces, however there are some differences between horizontally and vertically polarisations in the perfectly conducting roofs. In the Figure 3 , when the angle between the tips of the building roof is increased, for rough lossy roof and receiving antenna height is low, differences between horizontally and vertically polarizations can be observed more obviously, for example for $h_{r}=0 \mathrm{~m}$, when the angle between the tips of rough lossy roof is $95^{\circ}$, total loss is $30 \mathrm{~dB}$ for horizontally and vertically polarized waves. When the angle is $115^{\circ}$, total loss is $37 \mathrm{~dB}$ for horizontally polarized and $35 \mathrm{~dB}$ for vertical polarization as shown in the figures. When waves are horizontally polarized for the perfectly conducting roof, attenuation is greater than the others since the ray is parallel to the ground. While the angle between the tips of the roof is $95^{\circ}$, attenuation is $43 \mathrm{~dB}$.

\section{CONCLUSION}

Calculation of the propagation path loss between the transmitting and receiving antennas becomes possible with the contribution of the SD (Slope Diffraction) terms to the heuristic wedge diffraction 
coefficients. By changing interior angles of the tips of the building roof, for both polarizations, loss is increased as expected for rough lossy roof compared to perfectly conducting roof. Indeed, the building roofs are rough and lossy, in practice, diffracted field losses the derived formulation as given above, those are leads more realistic results for rough and lossy rough roofs.

\section{REFERENCES}

1. Kouyoumjian, R. G. and P. H. Pathak, "A uniform geometrical theory of diffraction for an edge in a perfectly conducting surface," Proc. IEEE, Vol. 62, 1448-1461, 1974.

2. McNamara, D. A., C. W. I. Pistorius, and J. A. G. Malherbe, Introduction to the Uniform Geometrical Theory of Diffraction, 471-500, Artech House, Inc., London, 1990.

3. Luebbers, R. J., "Finite conductivity uniform GTD versus knife edge diffraction in prediction of propagation path loss," IEEE Trans. Antennas Propagat., Vol. AP-32, No. 1, 70-76, 1984.

4. Luebbers, R. J., "Propagation prediction for hilly terrain using GTD wedge diffraction," IEEE Trans. Antennas Propagat., Vol. AP-32, No. 9, 951-955, 1984.

5. Luebbers, R. J., "A heuristic UTD slope diffraction coefficient for rough lossy wedges," IEEE Trans. Antennas Propagat., Vol. 2, 206-211, 1989.

6. Afacan, E. and E. Yazgan, "Modeling of mountains as wedges in communications," International Symposium on "Communication Theory \& Applications", Proc., Warwick-Ingiltere, 1991.

7. Afacan, E. and E. Yazgan, "Elliptic cylinder modelling of obstacles for low altitude radar systems," Journal of Electromagnetic Waves and Applications, Vol. 13, 237-258, 1999.

8. Kara, A. and E. Yazgan, "Modelling of shadowing loss due to huge non-polygonal structures in urban radio propagation," Progress In Electromagnetics Research B, Vol. 6, 123-134, 2008. 